\title{
Orientation tuning in human colour vision at detection threshold
}

\author{
SUBJECT AREAS: \\ COLOUR VISION \\ PERCEPTION
}

Received

26 November 2013

Accepted

18 February 2014

Published

5 March 2014

Correspondence and requests for materials should be addressed to K.T.M. (kathy.mullen@ mcgill.ca)

\author{
Mina Gheiratmand \& Kathy T. Mullen
}

McGill Vision Research, Department of Ophthalmology H4.14, 687 Pine Ave West, Montreal, Quebec, H3A 1A1 Canada.

We measure the orientation tuning of red-green colour and luminance vision at low $(0.375 \mathrm{c} / \mathrm{deg})$ and $\mathrm{mid}$ $(1.5 \mathrm{c} / \mathrm{deg})$ spatial frequencies using the low-contrast psychophysical method of subthreshold summation. Orientation bandwidths of the underlying neural detectors are found using a model involving Minkowski summation of the rectified outputs of a bank of oriented filters. At $1.5 \mathrm{c} / \mathrm{deg}$, we find orientation-tuned detectors with similar bandwidths for chromatic and achromatic contrast. At $0.375 \mathrm{c} / \mathrm{deg}$, orientation tuning is preserved with no change in bandwidth for achromatic stimuli, however, for chromatic stimuli orientation tuning becomes extremely broad, compatible with detection by non-oriented colour detectors. A non-oriented colour detector, previously reported in single cells in primate V1 but not psychophysically in humans, can transmit crucial information about the color of larger areas or surfaces whereas orientation-tuned detectors are required to detect the colour or luminance edges that delineate an object's shape.

he processing of shape and form begins with the encoding of local orientation information in the visual scene by arrays of neural mechanisms selective for different orientations. For luminance vision, numerous psychophysical studies have demonstrated the presence of orientation tuning in human vision, based on measurements of masking ${ }^{1-5}$, adaptation ${ }^{6-8}$, orientation discrimination ${ }^{9}$, or subthreshold summation ${ }^{5,10}$. Far fewer psychophysical studies have investigated orientation selectivity for colour vision, and have used adaptation $^{11}$, masking ${ }^{12,13}$ or orientation discrimination ${ }^{14}$.

Curiously, studies using the classic method of overlay masking in colour vision, which employ high contrast sinewave masks, have found orientation tuning functions that are flat ${ }^{12}$, or broadly tuned ${ }^{13}$. These relatively flat masking functions occur even though there is good evidence from other sources to support the presence of orientation tuning in colour vision ${ }^{14-16}$. It has been proposed that high contrast masks flatten the measured orientation tuning functions because they activate a mechanism of gain control, which suppresses sensitivity evenly across all orientations. This effect becomes confounded with the orientation tuning of the underlying sensory detectors ${ }^{3,17}$ making it difficult to separate the two. In comparison, a relative advantage of the psychophysical method of subthreshold summation is that it uses low, near threshold contrasts and therefore avoids the suppressive effects of contrast gain control, potentially allowing more specific estimates of the underlying detector bandwidths.

Our recent experiments using subthreshold summation have found evidence for two different types of colour mechanism revealed at detection threshold ${ }^{18}$. At low spatial frequencies, significant levels of subthreshold summation were found between superimposed orthogonal chromatic gratings, supporting the presence of very broadly tuned colour mechanisms, whereas at higher spatial frequencies, the cross orientation summation was lost, indicating the presence of orientation tuning. On the basis of these results, Gheiratmand, et al. ${ }^{18}$ proposed that colour vision has an isotropic mechanism that determines threshold at low spatial frequencies particularly for monocular stimuli, switching to an orientation-tuned response at higher spatial frequencies.

Here, we further develop the subthreshold summation technique to measure full orientation tuning curves for human colour vision at two different spatial frequencies, and for luminance vision under equivalent conditions. We derive estimates of the orientation bandwidth of the underlying chromatic responses based on measurements of how the amount of subthreshold summation declines as the orientation difference between two superimposed, sinusoidal gratings increases from 0 to 90 degs. Although it was initially thought that the tuning of this response is determined directly by the orientation bandwidths of the underlying sensory detectors ${ }^{19,20}$, we now know that the response tuning is also influenced by the orientation bandwidths of the visual stimuli and the summation across space of the neural responses to the stimuli ${ }^{5,9,21,22}$. Thus in order to extract the bandwidths of the underlying neural detectors from the response tuning functions, we use a modeling approach to take into account the effect of stimulus bandwidth and spatial summation. 
a.
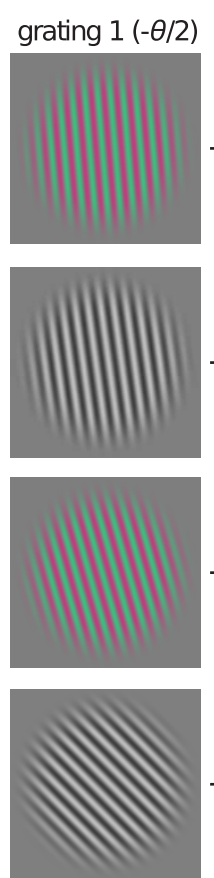

$\mathrm{SF}=1.5 \mathrm{c} / \mathrm{deg}$
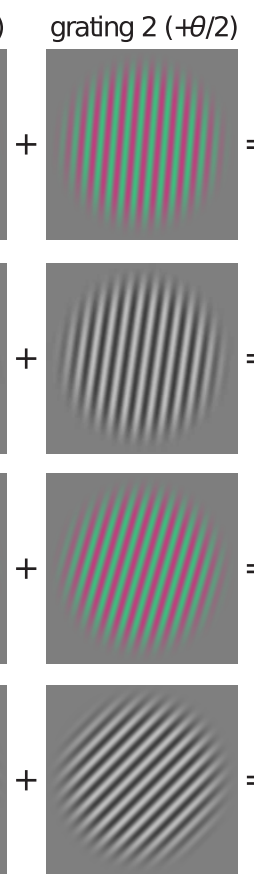

$=$ plaid $(\theta)$
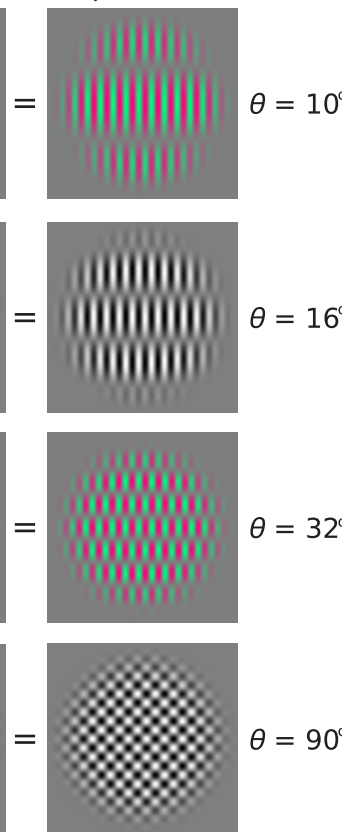

$\theta=90^{\circ}$

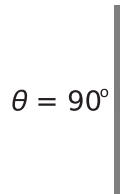

b.

grating $1(-\theta / 2)$
$\mathrm{SF}=0.375 \mathrm{c} / \mathrm{deg}$
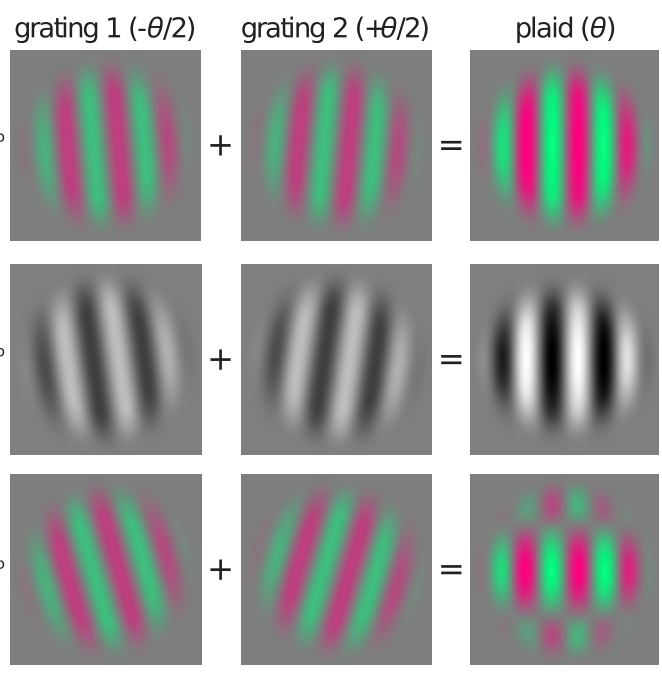
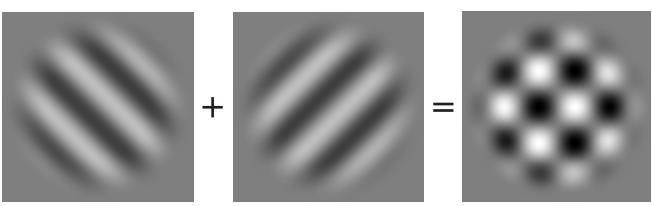

Figure 1 | Illustration of example stimuli. Examples of the red-green and achromatic component gratings and plaid stimuli at (a) $1.5 \mathrm{c} /$ deg and (b) $0.375 \mathrm{c} / \mathrm{deg}$, shown at high contrasts for illustrative purposes. Gratings are shown at $50 \%$ contrast.

In this paper, we first measure subthreshold summation as a function of the increasing orientation difference between two superimposed sinusoidal gratings (the response tuning functions), using the stimuli illustrated in Figure 1. We obtain these functions for luminance and colour contrast at two different stimulus spatial frequencies and fit them with von Mises functions to quantify their shapes and bandwidths. We then develop a model of the response functions to determine estimates of the orientation bandwidths of the underlying detectors using a spatial probability summation rule between outputs of a bank of orientation tuned filters. In particular, we aim to determine whether there is significant broadening in orientation tuning for lower spatial frequencies. In addition to a model of orientation tuning, we also employ a simple isotropic model, which lacks all orientation tuning and relies on a nonlinear neural summation.

At a mid spatial frequency $(1.5 \mathrm{c} / \mathrm{deg})$, we find evidence for orientation-tuned detectors that have similar bandwidths for isoluminant colour and achromatic stimuli. At a low spatial frequency $(0.375 \mathrm{c} / \mathrm{deg})$, however, distinctly different results were obtained: the orientation tuning curves for chromatic stimuli become very broad and are compatible with isotropic detectors, whereas for achromatic stimuli, orientation tuning is preserved with no change in bandwidth of the underlying detectors. These results support the presence of two types of spatial detector in colour vision: an orientation-tuned detector that can extract information about chromatic edges and contours in the visual scene, and a non-oriented detector, relatively more sensitive at low spatial frequencies, better suited to the representation of chromatic 'blobs' or larger areas of colour.

\section{Results}

Orientation tuning for colour and luminance contrast: mid versus low spatial frequencies. Figure 2 shows orientation tuning curves for two spatial frequencies, a mid spatial frequency of $1.5 \mathrm{c} / \mathrm{deg}$ (Fig. 2a) and a low spatial frequency of $0.375 \mathrm{c} / \mathrm{deg}$ (Fig. 2b), both viewed monocularly. Results are shown for chromatic (red circles) and achromatic stimuli (black triangles). The different panels show the results for three different subjects with the average results of all three subjects given in the top panel. When the two gratings are cooriented, or very closely oriented, the summation ratio (SR) is highest, at around a factor of $2(6 \mathrm{~dB})$, which is as expected from the physical combination of two identical co-oriented stimuli. As the orientation difference increases, however, the summation ratio falls to reveal the orientation tuning of the function.

A three-way mixed design ANOVA (2 (chromaticity) $\times 6$ (orientation $) \times 2$ (spatial frequency) $)$ showed a significant main effect of orientation: $F(5,20)=77.563, p<0.001$. Average summation ratio decreases as relative orientation increases. There are clear differences in the results for colour and achromatic stimuli that depend on the spatial frequency of the stimuli. The interaction between chromaticity and spatial frequency was significant: $F(1,4)=8.62, p=0.043$. The average summation ratio is similar for colour and achromatic stimuli at $1.5 \mathrm{c} / \mathrm{deg}$, but higher for colour stimuli compared to achromatic at $0.375 \mathrm{c} / \mathrm{deg}$. The main effect of spatial frequency was significant: $F(1,4)=18.10, p=0.013$. A Bonferroni posthoc test showed that the average summation ratio at $0.375 \mathrm{c} / \mathrm{deg}$ is significantly higher than $1.5 \mathrm{c} / \mathrm{deg}(p=0.013)$. The main effect of chromaticity was also significant: $F(1,4)=13.62, p=0.021$, with the average summation ratio being higher for the colour condition compared to achromatic (Bonferroni posthoc test: $p=0.021$ ). Both spatial frequency and chromaticity effects are driven by the higher summation ratios found for the colour compared to achromatic stimuli at $0.375 \mathrm{c} / \mathrm{deg}$. The interaction between spatial frequency and orientation was significant: $F(5,20)=5.873, p=0.002$, showing that summation ratio is affected differently by orientation for the two different spatial frequency conditions.

At $1.5 \mathrm{c} / \mathrm{deg}$ (Fig. 2a), summation decreases for both colour and achromatic stimuli as the orientation difference between the two component gratings increases, dropping to approximately half by 15 degs, and reaching minimal values at larger orientation differences ( $>30$ degs). This indicates the presence of orientation-tuned detectors with constrained bandwidths that generate little or no response to stimuli of widely separated and orthogonal orientations. At 


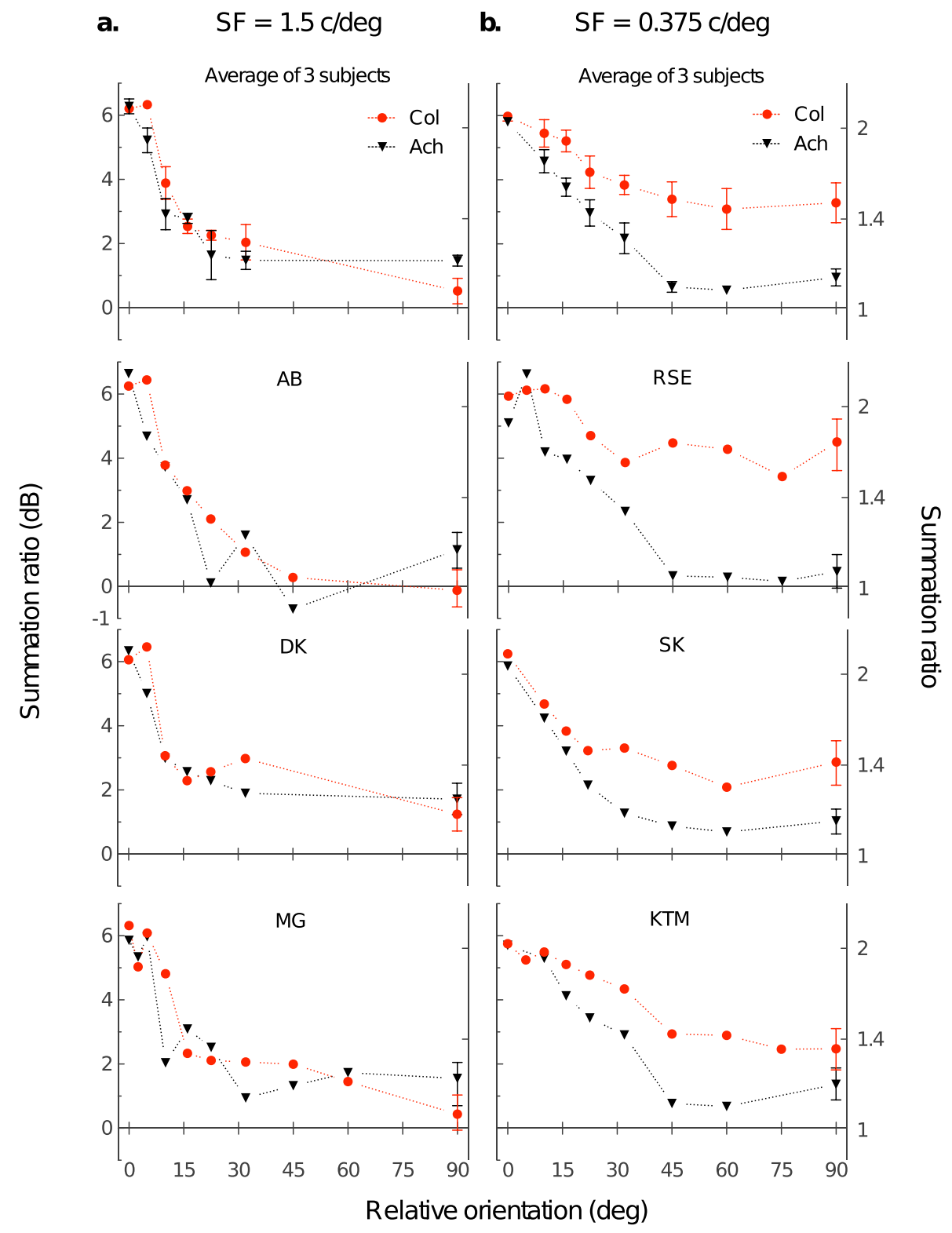

Figure $2 \mid$ Orientation tuning data for four experiment conditions. Orientation tuning curves for red-green (red circles) and achromatic (black triangles) stimuli at (a) $1.5 \mathrm{c} / \mathrm{deg}$ and (b) $0.375 \mathrm{c} / \mathrm{deg}$ for three subjects and their average. Summation ratio (right axis) also expressed in $\mathrm{dB}$ (left axis), is plotted as a function of relative orientation between the two gratings. Error bars show \pm s.e.m. for the averaged data. Sample error bars on individual data are the average s.d.'s across orientation, calculated using Gaussian error propagation.

this spatial frequency, the chromatic and achromatic tuning curves are very similar for all subjects $(\mathrm{AB}, \mathrm{MG}$, and $\mathrm{DK})$.

At $0.375 \mathrm{c} / \mathrm{deg}$ (Fig. 2b), the colour and achromatic tuning curves are substantially different. For the achromatic stimuli, summation ratios decline as the orientation difference increases, reaching relatively low values $(<1 \mathrm{~dB})$ by 45 degs. This suggests the presence of orientation-tuned detectors with constrained bandwidths. In contrast, for the chromatic stimuli, the orientation tuning curves are flatter and maintain a relatively high summation ratio (a factor of 1.4 or $3 \mathrm{~dB}$ ) even for large orientation differences (45-90 degs), suggesting that both component gratings are producing a response within a common neural mechanism with a very broad orientation bandwidth.

An earlier report measured subthreshold summation between pairs of orthogonal red-green gratings across two spatial frequencies in six subjects, with each subject completing both conditions ${ }^{18}$. A significant effect of spatial frequency was found, with higher summation ratios at lower spatial frequencies. This effect is compatible with the very broad orientation tuning seen for the chromatic, low spatial frequency stimuli in Figure 2.

In order to quantify and compare these orientation-tuning curves we fitted the individual and averaged functions with a one-dimensional von Mises function ${ }^{7,23,24}$. A von Mises function is the circular version of the Gaussian function and is appropriate for use with orientation-based data. It is also thought to be the best function to fit to neurophysiological orientation tuning data ${ }^{25}$. The range of the SR data varies between 1 and 2, so we use equation (1) to match the range of the experimental data:

$$
R=1+A \frac{V M}{\max (V M)}
$$

where $A$ is the scaling factor and $V M$ is the von Mises function: 


$$
V M(\theta)=\frac{e^{k \cos (2 \theta)}}{2 \pi I_{0}(k)}
$$

where $I_{0}(k)$ is a modified Bessel function kind one of order of zero, and $k$ is the concentration factor that determines the response bandwidth. The larger the $k$ value, the narrower is the bandwidth of the tuned curve. $k$ is a free parameter and is optimized using fminsearch routine in Matlab. $A$ is fixed to 1 for monocular conditions, because the maximum of monocular data is $\mathrm{SR}=2$ at $0 \mathrm{deg}$. The response bandwidth, Half Width at Half Height (HWHH), is calculated from the fitted $k$ parameter according to equation (3):

$$
\theta=0.5 \cos ^{-1}(1-\ln (2) / k)
$$

Figure $3 \mathrm{a}$ and $\mathrm{b}$ shows the von Mises fits to averaged colour (red circles) and achromatic (black triangles) data for $1.5 \mathrm{c} / \mathrm{deg}$ and $0.375 \mathrm{c} / \mathrm{deg}$ stimuli. The $k$ parameter, the bandwidth calculated from equation (3), and the goodness of fit measures (sum of squared errors, SSE, and R-squared) are shown in the inset of each panel. Fitted response bandwidths are: 15 degs and 12 degs for the chromatic and achromatic data at $1.5 \mathrm{c} / \mathrm{deg}$, respectively, and $47 \mathrm{degs}$ and 19 degs for average chromatic and achromatic data at $0.375 \mathrm{c} / \mathrm{deg}$, respectively (see Table 1). The fits to the data of individual subjects are available in the Supplementary Material (Fig. S1 and Table S1). Figure $3 \mathrm{c}$ plots the response bandwidths for the individual fits of the three subjects for each stimulus condition. At $0.375 \mathrm{c} / \mathrm{deg}$, response bandwidth for the colour stimulus is wider than for the achromatic by about 30 degs, whereas bandwidths at $1.5 \mathrm{c} / \mathrm{deg}$ are similar for colour and luminance vision with colour only 3 degs wider than luminance (Table 1).

Model estimates of neural detector orientation bandwidth. The shape of the orientation tuning response functions depends on three key factors: the orientation bandwidths of the visual stimuli, the orientation bandwidths of the underlying neural detectors, and the summation across space of the neural responses to the stimuli. In order to extract the bandwidths of the underlying neural detectors, we require a modeling approach to take into account the effect on the response tuning functions of stimulus bandwidth and spatial probability summation.

At $1.5 \mathrm{c} / \mathrm{deg}$ for both colour and achromatic contrast and at $0.375 \mathrm{c} / \mathrm{deg}$ for achromatic contrast, we find very little response summation between gratings with orientation differences $\geq 45$ degs (Fig. 2a and 2b achromatic), which indicates the presence of independent orientation-tuned filters for the detection of each oriented grating. This supports the use of a model in which outputs of the independent filters ${ }^{20}$ are combined based on a probabilistic [minimal] combination rule, a 'probability summation' model ${ }^{22,26-31}$. On the other hand, the wide tuning curves found for the chromatic stimulus at the low spatial frequency, with significant summation between orthogonal gratings, implies the presence of isotropic or very broadband detector mechanisms that respond to a wide range of stimulus orientations. Hence, a model capable of representing these data needs to encompass a generous combination rule that

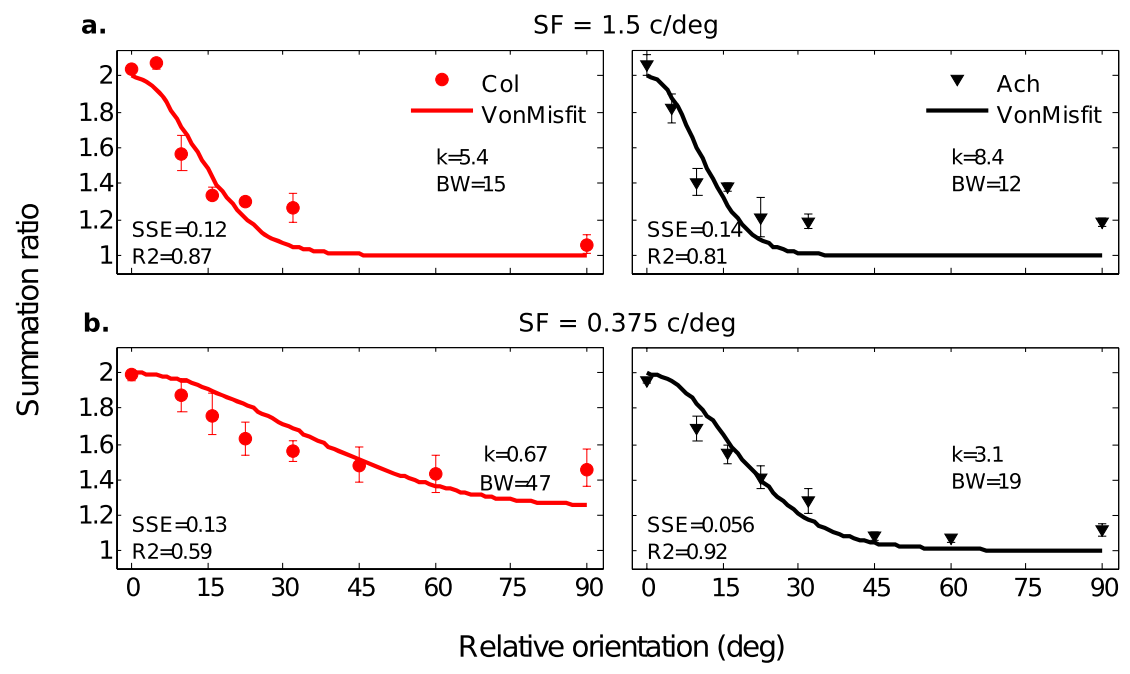

c.

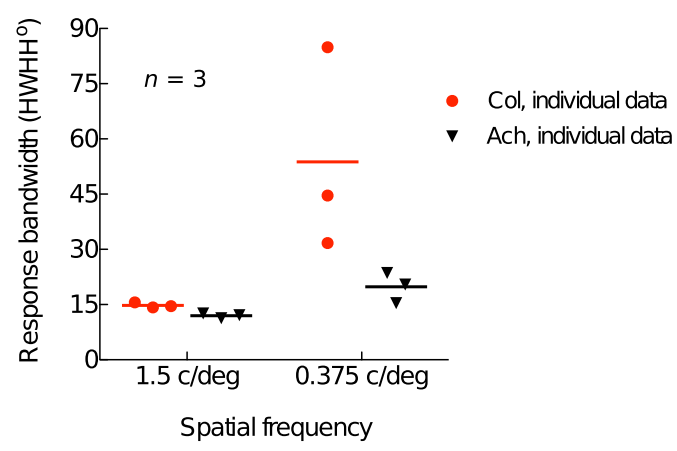

Figure 3 Fits to the response functions. Von Mises (equation (1)) fits to averaged colour (red circles) and achromatic (black triangles) data at (a) $1.5 \mathrm{c} / \mathrm{deg}$ and (b) $0.375 \mathrm{c} / \mathrm{deg}$. Estimate of the free parameter, $\mathrm{k}$, response bandwidth, and goodness of fit measures (SSE and $\left.\mathrm{R}^{2}\right)$ are shown in each panel. As bandwidth narrows, $\mathrm{k}$ increases. Error bars show \pm s.e.m. $\mathrm{R}^{2}$ indicates the proportion of variance in the data accounted for by the model fit, varying from 0 to 1. (c) Response bandwidths for three subjects for each stimulus condition and the average (solid line). (See Figure S1 and Table S1 in Supplementary Material for individual fits). 
Table 1 Response bandwidth and neural detector bandwidth estimates for different stimulus conditions, found from the descriptive Von Mises function fits and the tuned model fits respectively. Fits are found for each individual subject (average of the bandwidths \pm s.d. is presented for each condition) and for the average of the three subjects data and the results are shown in different rows

\begin{tabular}{|c|c|c|c|c|c|}
\hline & & \multicolumn{4}{|c|}{ Bandwidth (HWHH degs) } \\
\hline & & \multicolumn{2}{|c|}{$0.375 \mathrm{c} / \mathrm{deg}$} & \multicolumn{2}{|c|}{$1.5 \mathrm{c} / \mathrm{deg}$} \\
\hline & & Col & Lum & Col & Lum \\
\hline $\begin{array}{l}\text { Response (Von Mises) } \\
\text { Detector (Tuned model) }\end{array}$ & $\begin{array}{l}\text { Fit to Average of } 3 \text { subjects } \\
\text { Average BW of } 3 \text { subjects } \pm \text { s.d. } \\
\text { Fit to Average of } 3 \text { subjects } \\
\text { Average BW of } 3 \text { subjects } \pm \text { s.d. }\end{array}$ & $\begin{array}{l}47 \\
54 \pm 28 \\
62 \\
53 \pm 10.0\end{array}$ & $\begin{array}{l}19 \\
20 \pm 4 \\
9 \\
11 \pm 4.3\end{array}$ & $\begin{array}{l}15 \\
15 \pm 0.7 \\
16 \\
16 \pm 2.5\end{array}$ & $\begin{array}{l}12 \\
12 \pm 0.6 \\
12 \\
13 \pm 2.3\end{array}$ \\
\hline
\end{tabular}

has to occur within a neural mechanism, referred to as a 'neural combination' model ${ }^{18,32}$. Both models are discussed in detail in the following subsections. Their fits to our averaged data and estimates of bandwidths are shown in Figures 4 and 5.

a) Tuned model. We use a spatial probability summation model modified after Wilson and Bergen ${ }^{31}$, and Phillips and Wilson ${ }^{5}$ to find estimates of neural detectors bandwidth for each stimulus condition. The model uses a bank of Cartesian separable log Gabor filters ${ }^{5,17,33}$ centered at the same spatial frequency as the stimulus and oriented from 0 to 179 degs in steps of 10 degs. The response of the model is a probabilistic combination of the filters outputs over space and orientation, which is approximated by a Minkowski summation:

$$
R=\left\{\sum_{i=1}^{n} \sum_{x, y=1}^{M}\left|C . S(x, y) * F_{i}(x, y)\right|^{m}\right\}^{1 / m}
$$

where $C$ is component grating's contrast, $S(x, y)$ is the stimulus spatial pattern (grating or plaid), $M=267$ is the stimulus dimension in pixels, $F_{i}(x, y)$ is the $i$ th filter in the bank and $\mathrm{n}=18$ (180 degs/10 degs) is the number of filters in the bank, * indicates a 2 -dimensional convolution, and $m$ is the order of the Minkowski summation. $m$ is fixed at 4 , which has been consistently used in the literature for approximation of the probability summation rule ${ }^{27,31,34,35}$. For narrowly tuned filters, Minkowski summation becomes similar to a MAX operator for large $m$ values.
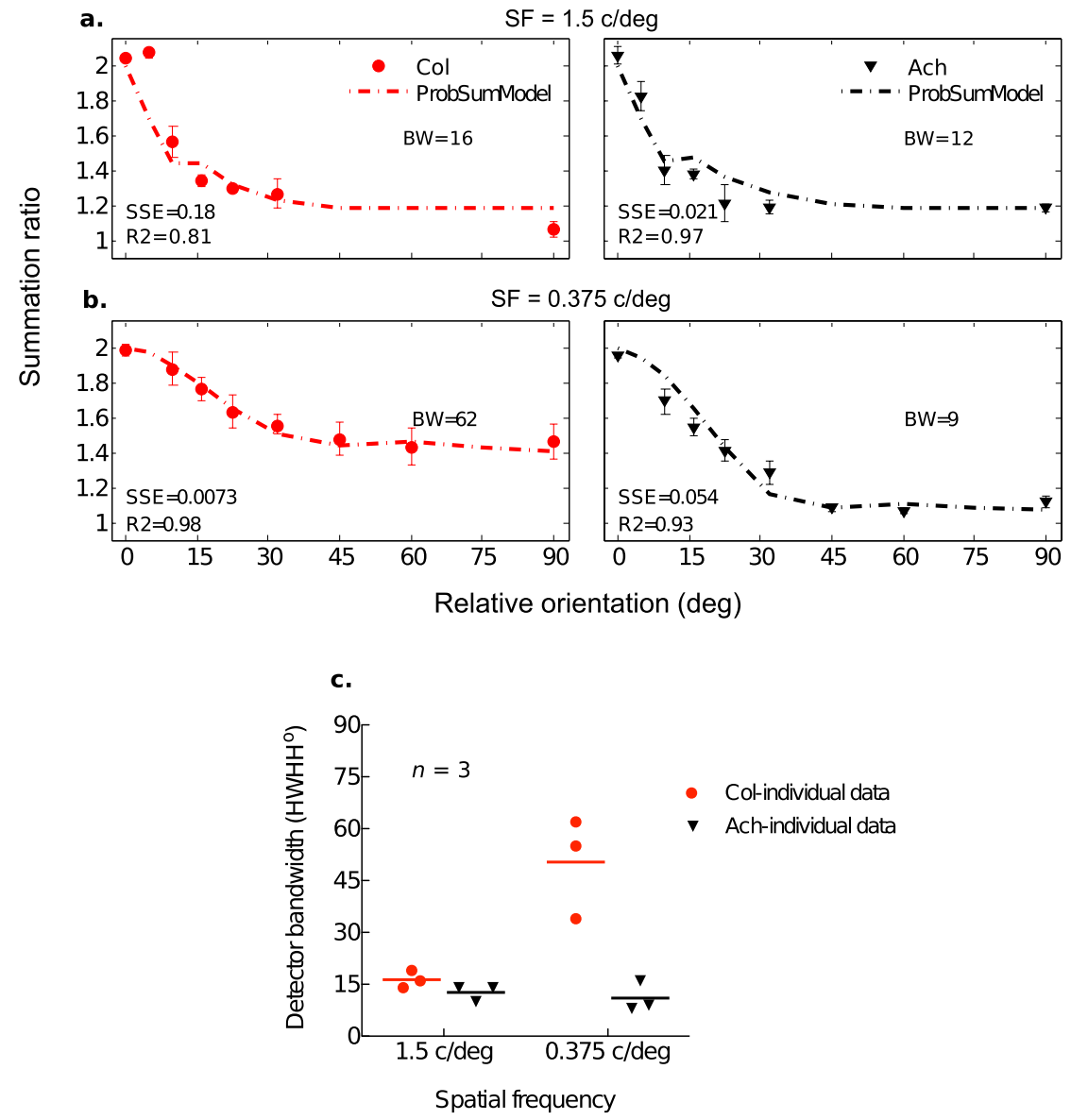

Figure $4 \mid$ Fits of the orientation-tuned model. Fits of the probability summation model to averaged colour (red circles) and achromatic (black triangles) data at (a) $1.5 \mathrm{c} / \mathrm{deg}$ and (b) $0.375 \mathrm{c} / \mathrm{deg}$. Orientation bandwidth estimates (HWHH in degs) from the model fit are shown in each panel. (c) Neural detector bandwidths for three subjects with the average (solid line) for each stimulus condition as marked. (See Figure S2 and Table S2 in Supplementary Material for individual fits). 

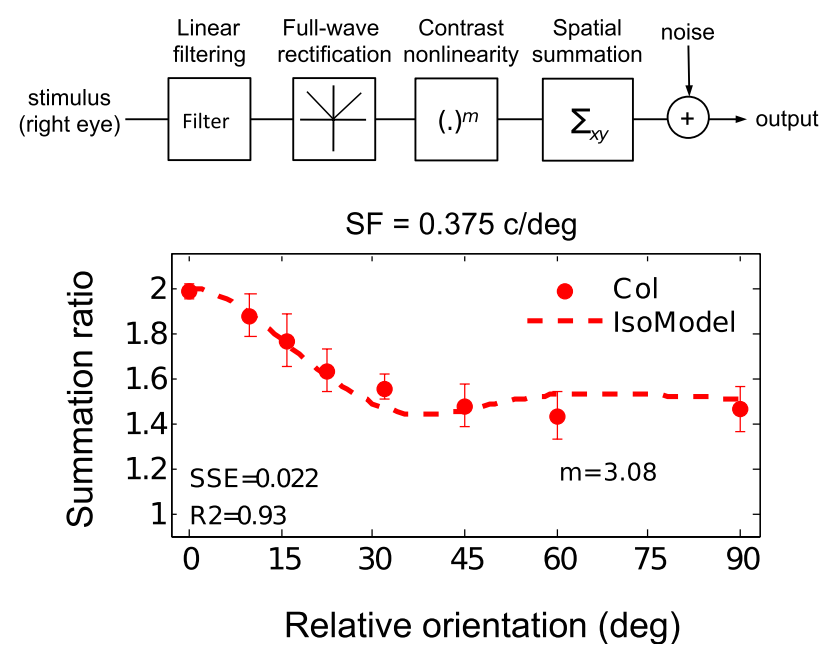

Figure 5 Fit of the Isotropic model. Fit of the isotropic model to the average colour data at $0.375 \mathrm{c} / \mathrm{deg}$, with model scheme illustrated above. The contrast nonlinearity exponent, $m$, estimated from the isotropic model and goodness of fit are shown in each panel. Error bars show \pm s.e.m. (See Figure S3 and Table S3 in Supplementary Material for individual fits.) The isotropic filter box in the schematic applies no spatial frequency or orientation constraints.

The spatial frequency bandwidth of the filters is set to 1.6 octaves for $\mathrm{SF}=1.5 \mathrm{c} / \mathrm{deg}$ and 2.2 octaves for $\mathrm{SF}=0.375 \mathrm{c} / \mathrm{deg}$, full width at half height, based on previous literature $\mathrm{e}^{36,37}$ and is similar for colour and luminance mechanisms ${ }^{11,38,39}$. The orientation bandwidth of the filters is the free parameter. We ascertained that steps finer than 10 degs for the filter bank were redundant. From equation (4) contrast at threshold $\left(\mathrm{C}_{\text {thresh }}\right)$ is found for the grating and plaid stimuli by setting $\mathrm{R}$ to an arbitrary value, $\mathrm{R}=1$ :

$$
C_{\text {thresh }}=\left\{\sum_{i=1}^{n} \sum_{x, y=1}^{M}\left|S(x, y) * F_{i}(x, y)\right|^{m}\right\}^{-1 / m}
$$

and summation ratio is calculated according to equation (9) in the Methods section.

The dashed curves in Figure $4 \mathrm{a}$ and $\mathrm{b}$ show the model fits to the averaged data for colour (red circles) and achromatic stimuli (black triangles) at $1.5 \mathrm{c} / \mathrm{deg}$ and $0.375 \mathrm{c} / \mathrm{deg}$, respectively. The bandwidth estimate, the fit error (SSE) and $\mathrm{R}^{2}$ goodness of fit measure are included in the inset of each graph. Linear SR data were used for fitting, and the best fit was chosen from a series of fits with filter orientation bandwidths varying from 5 to 62 degs in steps of $1 \mathrm{deg}$, based on the minimum root mean squared (RMS) error and highest $\mathrm{R}^{2}$.

At $1.5 \mathrm{c} / \mathrm{deg}$, orientation bandwidth (HWHH) estimates for the averaged data are similar for colour and achromatic contrast at 16 and 12 degs, respectively (Table 1). At $0.375 \mathrm{c} / \mathrm{deg}$, the fitted bandwidths were 9 degs for achromatic data averaged over three subjects and 62 degs for colour vision. (We note that 62 degs is the broadest possible bandwidth for our log Gabor filters). Figure $4 \mathrm{c}$ shows the filter bandwidths for the three subjects for different stimulus conditions, with averages given in Table 1. (Figure S2 and Table S2 of the Supplementary Material show the fits for individual subjects.)

The results show that the bandwidths of the colour detectors at $0.375 \mathrm{c} / \mathrm{deg}$ are extremely broad and considerably greater than the other three conditions, and the filters overlap, even at orthogonal orientations, to generate an SR of $1.4(\sim 3 \mathrm{~dB})$. This means there is considerable summation within a single neural mechanism, which responds to both component gratings. In the light of this, in the next section, we apply a different version of the model to the low spatial frequency chromatic data, which takes into account neural summation within a single isotropic detector ('neural summation model'). The same model configuration is obtainable if we replace the tuned filters in the probability summation model with isotropic filters. The resulting curve is shown by the thin dashed line in Supplementary Figure S3.

b) Isotropic Model. The significant summation at large plaid angles of 45 to 90 degs for the low spatial frequency colour data (Fig. 2b, red circles) signifies the presence of very broadband or isotropic mechanisms that respond to orthogonal orientations. We test the hypothesis of an isotropic neural detector by applying an 'isotropic model' (modified after Meese and Summers ${ }^{40}$, Meese ${ }^{33}$, and Gheiratmand, et al. ${ }^{18}$ ). Similar to an energy model ${ }^{41,42}$, this model involves an isotropic filter, full-wave rectification, non-linear contrast transduction and spatial summation as illustrated in Figure 5:

$$
R_{i s o}=\sum_{x, y=1}^{M}(C .|S(x, y)|)^{m}
$$

where $m$ is the contrast exponent order, and the other parameters are as for equation (4). The isotropic filter has no spatial tuning and is transparent. We can find the threshold of the gratings and the plaids by setting $R$ equal to an arbitrary value of 1 :

$$
C_{\text {thresh }}=\left(\sum_{x, y=1}^{M}|S(x, y)|^{m}\right)^{-1 / m}
$$

The exponent order, $m$, is the only free parameter in the model, which is optimized using fminsearch routine in Matlab.

Figure 5 shows the model fit to the averaged colour data at $0.375 \mathrm{c} /$ deg with the fit parameters included in the inset. The contrast exponent $m$ equals 3.08 for the average fit $(3.26 \pm 2.02$ s.d. averaged over three subjects), which is compatible with the range found for contrast nonlinearity in many other studies and the energy model ${ }^{29,33,40-42}$. The $m$ value is also compatible with Pelli ${ }^{43}$ approximation of contrast exponent, $m^{\prime}=\beta / 1.25$, where $\beta$ is the slope of the Weibull psychometric function fit to data. The slope averaged over all stimulus conditions and all subjects is $\beta=2.95 \pm 0.14 \mathrm{SE}$, and from there $m^{\prime}=2.36$. The use of an exponent determined directly from the measurements of the slopes of our psychometric functions ( $m=$ 2.36) also provides a good model fit to the data (Fig. S3), although we have used the best fitting exponent of $m=3.08$. The isotropic model provides a reasonable fit to colour data at $0.375 \mathrm{c} / \mathrm{deg}$ (RMSE $=0.05$ and $\mathrm{R}^{2}=0.93$ for the fit to the averaged data), supporting the plausibility of non-oriented colour mechanisms at low spatial frequencies. (Fits for individual subjects and parameter estimates are given in Figure S3 and Table S3 of the supplementary material.) We also fitted the isotropic model to the equivalent achromatic data at $0.375 \mathrm{c} / \mathrm{deg}$ for comparison purposes, but rejected it because it was a worse fit than the tuned model and failed to capture the key characteristics of the data, specifically the very low summation ratios for large plaid angles.

Note on response vs. neural detector bandwidths. It is interesting to note that stimulus-based effects may influence the response tuning, particularly for the low spatial frequencies that have fewer number of spatial cycles. For example for the chromatic stimuli at $0.375 \mathrm{c} / \mathrm{deg}$, we see some tuning of the response even in the absence of any tuning of the underlying neural detectors (Fig. 5). This reflects the decline of stimulus energy for different plaid patterns at small angles, where beating occurs between overlaid grating components and affects the result of spatial summation. The model fit to the empirical data is reflecting the fact that the psychophysical response is dependent on the stimulus energy. For the low spatial frequency achromatic stimuli (Fig. 4b), the response tuning is broader than the fitted underlying detectors (by about 10 degs), again reflecting the influence of the 
stimulus-based effects. For the higher spatial frequency, the response bandwidths are very similar to the neural detector bandwidths. These stimuli have more spatial cycles and narrower orientation tuning (in relation to the detector bandwidth) and the stimulus effects have less influence on the response tuning. Thus explicit models are required to relate the response tuning to the underlying detector bandwidths, given the stimuli used.

\section{Discussion}

We have used the classical psychophysical method of subthreshold summation to measure orientation tuning of the visual detectors underlying human colour vision at different spatial frequencies. We fit the response functions using a von Mises function since this function provides a good fit and a measurement of bandwidth using the fewest parameters. However, this function, or any other, is simply a description of the data and provides little insight into the visual mechanisms involved in generating the response. In order to determine the orientation tuning of the underlying sensory detectors, we use a model involving arrays of orientation and spatial frequency tuned filters whose outputs are combined across the visual extent of the stimulus using a Minkowski summation rule. This method has been used previously to determine orientation ${ }^{5}$ and spatial frequency tuning ${ }^{26,31}$ for achromatic stimuli. By fitting this model, we obtain an estimate of the orientation bandwidths of the underlying detectors. Since, in some cases the best fitting orientation tuning was very broad and was at the maximum that could be achieved, we also used an isotropic version of the model with no orientation tuning.

At low spatial frequencies, we find that the chromatic response function is much broader than the achromatic. We found that an isotropic model, which assumes no orientation tuning of the underlying detectors, provided a good fit to the chromatic response function. The orientation-tuned model could only provide a reasonable account of the data if we used very broad detector bandwidths of 62 degs (HWHH), which was the broadest possible bandwidth yielded from our log Gabor filters. Effectively, a very broadly tuned and an isotropic model are similar in that both predict significant summation between orthogonal gratings. There are differences, however, in the shapes of the underlying spatial filters, as illustrated in Figure 6. Figure $6 \mathrm{c}$ illustrates the spatial filter obtained from the tuned model fit to the chromatic data. The filter is highly elongated along the horizontal axis in order to render the broad orientation tuning. There is no physiological evidence, however, for the elongated detector of the type. Figure $6 \mathrm{e}$ and $\mathrm{f}$ illustrates the filters that may underlie the isotropic model, for which the constraints of orientation and spatial frequency tuning have been removed. Crucially, these filters are circularly symmetric, and may be low pass for chromatic spatial frequency (Fig. 6e), corresponding to type 1 or type 2 receptive fields as originally defined by Wiesel and Hubel ${ }^{44}$, or tuned to chromatic spatial frequency (Fig. 6f), so resembling a circularly symmetric dual opponent receptive field. There is plentiful evidence for these types of isotropic detectors in the primate cortex, as discussed further below (and see Shapley and Hawken ${ }^{45}$ for a review) ${ }^{46-49}$. Hence we conclude that an isotropic detector model provides more plausible account of the chromatic response at low spatial frequencies than a tuned model that requires an extreme filter bandwidth.

For the achromatic stimuli, the response functions are best fitted by a model with orientation-tuned detectors, and yield bandwidths of 9 degs, as illustrated in Figure 6d. The isotropic model could not properly fit the achromatic response tuning functions.

At the mid spatial frequency of $1.5 \mathrm{c} / \mathrm{deg}$, both chromatic and achromatic response functions show marked orientation tuning with the model fit indicating orientation-tuned detectors with only a small difference in bandwidth (16 degs for chromatic and 12 degs for achromatic). The presence of orientation tuning in colour vision, slightly broader than for achromatic contrast, is in agreement with previous studies using other methods ${ }^{11,13,14}$. Thus there is a clear

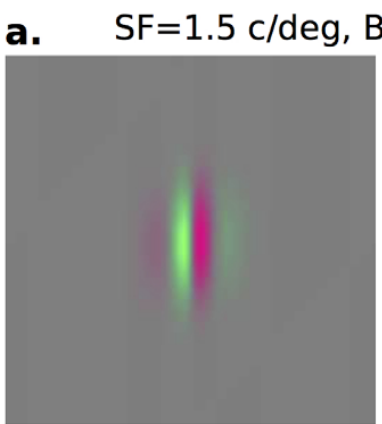

$\mathrm{BW}=16^{\circ}$

c. $\mathrm{SF}=0.375 \mathrm{c} / \mathrm{deg}, \mathrm{BWsf}=2.2$ octave

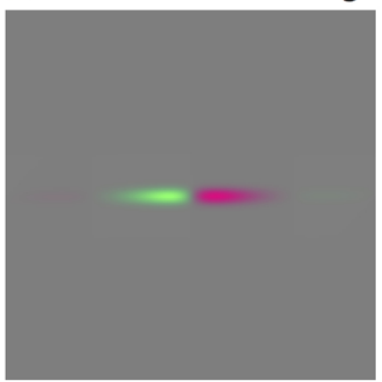

$\mathrm{BW}=62^{\circ}$

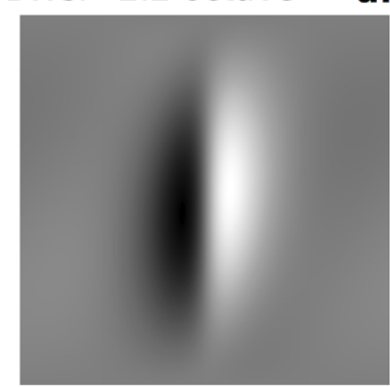

$\mathrm{BW}=9^{\circ}$

e.
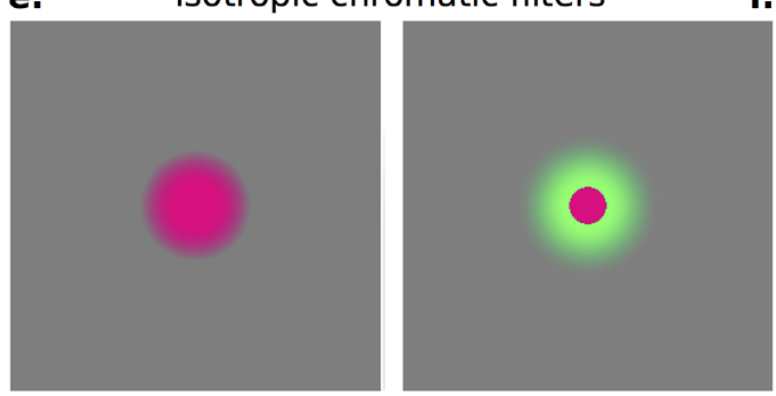

Figure 6 | Illustrations of the filter shapes underlying threshold detection. Representation of the optimal Log Gabor filter estimated from the model fits for the average (a) colour (red-green) and (b) achromatic (grey) data at the mid spatial frequency. (c-d) optimal Log Gabor filter estimates for the colour and achromatic data at the low spatial frequency, respectively. (e-f) possible neural detectors underlying the isotropic model: (e) an isotropic (circularly symmetric) single opponent detector, low-pass for chromatic spatial frequency, and (f) a band pass isotropic dual opponent detector tuned to chromatic spatial frequency. Red and green parts represent the stimulus color that the detector best responds to within its receptive field.

effect of spatial frequency on the orientation tuning of colour contrast, with a switch from orientation tuned to isotropic detectors at low spatial frequencies, but for achromatic contrast we found no evidence for any increase in bandwidth as spatial frequency declined.

Previous masking studies using achromatic stimuli have reported that orientation bandwidth increases as spatial frequency decreases ${ }^{1,5,50}$, something that we do not find in our study. Meese and Holmes ${ }^{17}$ and Cass, et al. ${ }^{3}$ have argued that the suppressive effect of contrast normalization, which is thought to increase at lower spatial frequencies, may account for this effect, and argued for constant underlying detector bandwidths across spatial frequency of around 20 degs. However, Cass, et al. ${ }^{3}$ only measured response tuning, providing no model of the detector tuning, and so estimated bandwidths are likely to be broader due to stimulus-based effects. 
The use of very low contrasts in the subthreshold summation method allows us to measure the orientation tuning of the neural detectors more directly by avoiding the suppressive effect of contrast normalization inherent in the use of high contrast masking or adaptation methods. Contrast normalization, revealed as the perceptual effect called "cross orientation masking", elevates thresholds across all orientations, broadening the orientation tuning of the masking ${ }^{3,17}$. The effect of cross orientation masking is stronger in colour than luminance vision ${ }^{12,51}$ and acts to obscure the orientation tuning of the underlying colour detectors in masking experiments; for example, Medina and Mullen ${ }^{12}$ found no orientation tuning in the chromatic response functions using masking. For both chromatic and achromatic contrast, our bandwidth estimates at $1.5 \mathrm{c} / \mathrm{deg}(16$ and 12 degs, for colour and luminance respectively) are narrower than those found previously from masking experiments, which are in the range of 26-30 degs for colour and 22-24 degs for luminance contrast at a mid-spatial frequency ${ }^{5,13,14}$. This difference may be accounted for by the isotropic effects of contrast normalization in masking, as well as by other differences in the stimuli or models used.

Our results reveal the presence of non-oriented detectors sensitive to low spatial frequencies in human colour vision for monocular viewing conditions. They support our earlier report based on measuring subthreshold summation between pairs of orthogonal gratings $^{18}$. While the previous study suggested the presence of isotropic detectors at low spatial frequencies switching to tuned detectors at higher spatial frequencies, the experiments described here yield the complete tuning functions and provide models for orientation tuned versus isotropic detection that allow bandwidth estimates for each condition.

The isotropic detectors that we find are likely to reflect the activity of non-oriented, circularly symmetric chromatic neurons in primate cortical areas V1 and V2. In colour vision, the most commonly reported non-oriented cortical neurons are type 2 , which have circularly symmetric receptive fields with single cone opponency. These neurons are spatially low pass for colour contrast, responding best to low spatial frequencies, and are thought to form around $10 \%$ of the population in primate $\mathrm{V} 1^{45,47,52}$. They have been called "area responsive" because they will produce strong responses to the interior regions of coloured objects or figures and are not edge sensitive. Hence, they can potentially transmit crucial information about the colour of a surface. Circularly symmetric dual opponent neurons have also been reported in $\mathrm{V}^{46}$. Such cells are unselective for orientation, but have spatial selectivity, and have been typically investigated using "blobs" of colour on a contrasting background. These neurons, if tuned to a low spatial frequency, could also underlie the isotropic response to our low spatial frequency chromatic stimuli and provide information about larger regions of colour against a background. The presence of isotropic dual opponent neurons is controversial, however ${ }^{45}$, and it has been suggested that they may in fact be the type 2 neurons above. Most dual opponent neurons are orientation tuned and edge-responsive and form around $30 \%$ of the population in V1, making them ideal candidates for underlying the orientation-tuned chromatic response that determines threshold at the mid-spatial frequency ${ }^{45,47,49,53}$. Although it is unknown at what stage psychophysical threshold is determined, the colour selective non-oriented and orientation-tuned neural populations in V1 have sufficient sensitivity to be plausible candidates for psychophysical threshold ${ }^{54}$. In conclusion, we suggest that the psychophysical, non-oriented chromatic detector that we find is likely to be involved in providing information about the colour of a larger area or surface, whereas the orientation-tuned detectors, which are selective for colour edges and contours, are likely to be involved in delineating shape or form. In further support of this distinction of roles, evidence is emerging for distinct visual areas found in extra striate (occipitotemporal) cortex that respond differentially to the shape of an object as opposed to its surface properties (colour and texture) ${ }^{55}$, with colour vision providing input into both these types of function.

\section{Methods}

Apparatus. Stimuli were generated using a ViSaGe video-graphics card (Cambridge Research Systems, Kent, UK) with 14-bit contrast resolution, programmed in Matlab and were displayed on a CRT colour monitor (Iiyama Vision Master Pro 513, Iiyama Corporation) with a resolution of $1024 \times 768$ and a frame refresh rate of $120 \mathrm{~Hz}$. The monitor was gamma corrected using an OptiCal photometer (CRS) and the VSG Desktop software. The spectral radiances of the red, green, and blue phosphors of the monitor were calibrated using a PR-645 Spectrascan spectroradiometer (Photo Research Inc, Chatsworth, CA, USA). The CIE $1931 \mathrm{x}-\mathrm{y}$ chromaticity coordinates for the red, green, and blue phosphors outputs were $(x=0.624, y=0.335),(x=0.293$, $y=0.608)$ and $(x=0.147, y=0.073)$, respectively. The background was achromatic with a mean luminance of $43 \mathrm{~cd} / \mathrm{m}^{2}$ at the screen centre. All stimuli were viewed through a mirror stereoscope in a dimly lit room with an optical distance of $58 \mathrm{~cm}$.

Observers. Six observers participated in the study, the two authors (MG and KTM) and four naïve subjects with previous training in psychophysics $(\mathrm{AB}, \mathrm{DK}, \mathrm{SK}$, and RSE). Each subject completed at least two of the four stimulus conditions, colour and achromatic contrast at one of the two spatial frequencies. All subjects had normal or corrected-to-normal visual acuity and normal colour vision, assessed with the Farnsworth-Munsell 100 Hue test. The experiments were performed in accordance with the Declaration of Helsinki and approved by the institutional ethics committee of McGill University Health Centre. An information consent form was signed by each of the subjects.

Stimuli. Stimuli were achromatic or isoluminant red-green sine-wave gratings (phase $=0$ ) presented alone or as pairs of gratings of the same spatial frequency overlaid to form a plaid, as illustrated in Figure 1. Stimuli were sinewave gratings of 0.375 or $1.5 \mathrm{c} / \mathrm{deg}$ and were displayed in a circular patch (10 degs in diameter) with edges contrast enveloped with a spatial raised cosine ( $2.5 \mathrm{degs})$. Stimuli were static and presented in a temporal Gaussian envelop with a sigma of $125 \mathrm{~ms}$. The two component gratings were generated independently and were combined in alternative monitor frames at $120 \mathrm{~Hz}$ using frame interleaving with a frame-cycling option in software. In the experiments, the orientation difference $(\theta)$ between the grating pairs forming the plaid was varied between 0 and 90 degs, as illustrated in Figure 1. Component gratings were positioned $\pm \theta / 2$ relative to the vertical axis to avoid any potential oblique effect ${ }^{56}$. Stimuli were viewed monocularly with right eye and the left eye viewed a uniform field of the same mean luminance.

Colour space. Stimuli are represented in a three-dimensional cone contrast colour space $^{57,58}$ in which each axis is defined by the incremental stimulus intensity for each cone type to a given stimulus normalized by the respective intensity of the fixed adapting white background. Cone excitations for the L-, M-, and S-cones were calculated using the Smith and Pokorny cone fundamentals. A linear transform was calculated to specify the required phosphor contrasts of the monitor for given cone contrasts.

Stimulus contrast is defined as the vector length in cone contrast units $\left(C_{C}\right)$ :

$$
C_{C}=\sqrt{\left(L_{C}\right)^{2}+\left(M_{C}\right)^{2}+\left(S_{C}\right)^{2}}
$$

where $L_{c}, M_{c}$, and $S_{c}$ represent the $\mathrm{L}, \mathrm{M}$, and $\mathrm{S}$ cone-contrast for the L, M, and $\mathrm{S}$ cones. This metric differs by a factor of $\sqrt{3}$ from the Michelson luminance contrast. Chromatic stimuli were isoluminant and activated the $\mathrm{L} / \mathrm{M}$ cone opponent pathway. The stimulus vector direction was (L- $\alpha \mathrm{M})$, where $\alpha$ is a numerical constant obtained at isoluminance. Achromatic stimuli had the vector direction $(L+M+S)$. For each subject, the isoluminant point was determined using a minimum motion experiment as previously described ${ }^{18}$. The subject viewed a drifting Gabor whose spatial frequency matched that used in the main experiment and a method of adjustment was used to determine the L:M cone ratio (value of $\alpha$, above) at which the subject perceived motion to be at a minimum. This was repeated 10-20 times for each eye, and binocularly, and the average was taken as isoluminance. We did not find a difference between the two eyes in any subject. The $\alpha$ value at isoluminance was: $4,4,11.4,2.1,1$, and 3.4 for subjects DK, AB, MG, RSE, SK, and KTM respectively.

Protocols. For each summation experiment, detection thresholds were obtained for a left oblique grating, a right oblique grating, and the plaid composed of the superimposition of the two gratings. We used 9 orientation differences for the pairs of component gratings and their corresponding plaids ( $\pm \theta / 2)$ (see Stimuli): $0, \pm 5, \pm 8$, $\pm 11.25, \pm 16, \pm 22.5, \pm 30, \pm 35.5$, and \pm 45 degs. We measured detection thresholds using a temporal two-alternative-forced choice (2AFC) method of constant stimuli, in which one interval contained the stimulus and the other was blank (mean luminance). Each stimulus interval was $500 \mathrm{~ms}$ in duration, cued with a beep, with an inter-stimulus interval of $400 \mathrm{~ms}$. Subjects had to choose in which interval, first or second, they detected the stimulus. Auditory feedback was provided. Detection thresholds were derived from fits of the psychometric functions plotting per cent correct for stimulus detection as a function of cone contrast (achromatic or colour), based on measurements for at least six different contrasts with 90 to 120 trials per contrast. Data collection was made over several sittings with presentations of the two gratings and their combination ordered in a balanced block design. Psychometric 
functions were fitted with a standard Weibull function with the chance level set to 0.5 , the lapse rate constrained to 0.02 , and the parameters for contrast detection threshold corresponding to $81.6 \%$ correct detection and the slope of the function were extracted. Fitting was done using the psignifit toolbox for Matlab ${ }^{59}$. The variability of threshold and slope parameter estimates was found using the bootstrap method based on 4,999 simulations ${ }^{60}$.

We did not find systematic differences between the detection thresholds for the component gratings of different orientations. Typically, we combined the psychometric data for all component-grating orientations, yielding 110-480 trials per contrast level of the psychometric function. In one subject (SK), however, full psychometric functions were collected on each component grating for the colour condition, and data was only combined across the two components, yielding at least 80 trials per contrast level. In another subject (KTM), we pooled the grating data for grating pairs of similar orientations so increasing the number of trials per threshold estimate (at least 140 trials per contrast level). Psychometric functions for the plaids were based on 90 to 100 trials per contrast level, yielding a total of 540-600 trials per psychometric function.

Analysis. To quantify the amount of visual response summation between the two combined component gratings we calculated a summation ratio, which is defined as the ratio of the contrast at detection threshold for a component grating presented alone $\left(\right.$ Thresh $\mathrm{Grat}_{\text {t }}$ ) to its contrast in the plaid when plaid is at detection threshold (Thresh Plaid $_{\text {): }}$

$$
S R=\frac{\text { Thresh }_{\text {Grat }}}{\text { Thresh }_{\text {Plaid }}}
$$

Both component gratings composing a plaid have equal contrast. An SR of 2 shows a linear summation between responses to two component gratings and is expected for the case of monocular plaid presentation, where the two component gratings interleaving in successive frames are co-oriented. A ratio of near 1 indicates the absence of neural summation and is found, for instance, for a luminance orthogonal plaid at a high spatial frequency ${ }^{18}$. In this case, while physical input to the visual system has been increased by the combination of two gratings into a plaid compared to a single grating, visual sensitivity does not improve. This indicates involvement of independent orientation tuned mechanisms in the detection of the compound stimulus $^{21}$. Notwithstanding, a small improvement is still expected even for orthogonal stimuli and observed because of higher probability of detection in presence of two stimuli compared with one (classically referred to as probability summation). A summation ratio between 1 and 2 signifies some sort of neural summation that can be assessed by employment of a model.

Orientation tuning curves. By measuring summation ratio as a function of plaid angle from 0 to 90 degs we reveal the orientation tuning of the response. These response curves are measured for four different stimulus conditions: colour and achromatic contrast at low $(0.375 \mathrm{c} / \mathrm{deg})$ and $\mathrm{mid}(1.5 \mathrm{c} / \mathrm{deg})$ spatial frequencies presented monocularly. The bandwidths of the response tuning are found by fitting a von Mises function to the orientation tuning curves (see Results). To find estimates of the bandwidths of the underlying neural detectors we employ a modeling approach, as described in the Results.

1. Blake, R. \& Holopigian, K. Orientation selectivity in cats and humans assessed by masking. Vision Res 25, 1459-1467 (1985).

2. Campbell, F. W. \& Kulikowski, J. J. Orientational selectivity of the human visual system. J Physiol 187, 437-445 (1966).

3. Cass, J., Stuit, S., Bex, P. \& Alais, D. Orientation bandwidths are invariant across spatiotemporal frequency after isotropic components are removed. $J$ Vis 9,17 11-14 (2009).

4. Foley, J. M. Human luminance pattern-vision mechanisms: masking experiments require a new model. J Opt Soc Am A 11, 1710-1719 (1994).

5. Phillips, G. C. \& Wilson, H. R. Orientation bandwidths of spatial mechanisms measured by masking. J Opt Soc Am A 1, 226-232 (1984).

6. Blakemore, C. \& Nachmias, J. The orientation specificity of two visual aftereffects. J Physiol 213, 157-174 (1971).

7. Cass, J., Johnson, A., Bex, P. J. \& Alais, D. Orientation-specificity of adaptation: isotropic adaptation is purely monocular. PLoS One 7, e47425, doi:10.1371/ journal.pone.0047425 (2012).

8. Movshon, J. A. \& Blakemore, C. Orientation specificity and spatial selectivity in human vision. Perception 2, 53-60 (1973).

9. Thomas, J. P. \& Gille, J. Bandwidths of orientation channels in human vision. JOpt Soc Am 69, 652-660 (1979).

10. Kulikowski, J. J., Abadi, R. \& King-Smith, P. E. Orientational selectivity of grating and line detectors in human vision. Vision Res 13, 1479-1486 (1973).

11. Bradley, A., Switkes, E. \& De Valois, K. Orientation and spatial frequency selectivity of adaptation to color and luminance gratings. Vision Res 28, 841-856 (1988).

12. Medina, J. M. \& Mullen, K. T. Cross-orientation masking in human color vision. $J$ Vis 9, 20 1-16 (2009).

13. Pandey Vimal, R. L. Orientation tuning of the spatial-frequency-tuned mechanisms of the red-green channel. J Opt Soc Am A 14, 2622-2632 (1997).
14. Beaudot, W. H. \& Mullen, K. T. Orientation selectivity in luminance and color vision assessed using 2-d band-pass filtered spatial noise. Vision Res 45, 687-696 (2005).

15. Webster, M. A., De Valois, K. K. \& Switkes, E. Orientation and spatial-frequency discrimination for luminance and chromatic gratings. J Opt Soc Am A 7, 1034-1049 (1990).

16. Wuerger, S. M. \& Morgan, M. J. Input of long- and middle-wavelength-sensitive cones to orientation discrimination. J Opt Soc Am A 16, 436-442 (1999).

17. Meese, T. S. \& Holmes, D. J. Orientation masking and cross-orientation suppression (XOS): implications for estimates of filter bandwidth. J Vis 10, 9 1-20 (2010).

18. Gheiratmand, M., Meese, T. S. \& Mullen, K. T. Blobs versus bars: psychophysical evidence supports two types of orientation response in human color vision. $J$ Vis 13, 2 1-13 (2013).

19. Kulikowski, J. J. \& King-Smith, P. E. Spatial arrangement of line, edge and grating detectors revealed by subthreshold summation. Vision Res 13, 1455-1478 (1973).

20. Sachs, M. B., Nachmias, J. \& Robson, J. G. Spatial-frequency channels in human vision. J Opt Soc Am 61, 1176-1186 (1971).

21. Graham, N. V. S. Visual Pattern Analyzers. (Oxford University Press, 1989).

22. Nachmias, J. in Computational Models of Visual Processing (eds Landy, M. S. \& Movshon, J. A.) (The MIT Press, 1991).

23. Ringach, D. L. Spatial structure and symmetry of simple-cell receptive fields in macaque primary visual cortex. J Neurophysiol 88, 455-463 (2002).

24. Mardia, K. V. \& Zemroch, P. J. Algorithm AS 86: The von Mises distribution function. J Roy Stat Soc C-App 24, 268-272 (1975).

25. Swindale, N. V. Orientation tuning curves: empirical description and estimation of parameters. Biol Cybern 78, 45-56 (1998).

26. Bergen, J. R., Wilson, H. R. \& Cowan, J. D. Further evidence for four mechanisms mediating vision at threshold: sensitivities to complex gratings and aperiodic stimuli. J Opt Soc Am 69, 1580-1587 (1979).

27. Quick, R. F., Jr. A vector-magnitude model of contrast detection. Kybernetik 16, 65-67 (1974)

28. Robson, J. G. \& Graham, N. Probability summation and regional variation in contrast sensitivity across the visual field. Vision Res 21, 409-418 (1981).

29. Watson, A. B. \& Ahumada, A. J., Jr. A standard model for foveal detection of spatial contrast. J Vis 5, 717-740 (2005).

30. Watson, A. B., Thompson, P. G., Murphy, B. J. \& Nachmias, J. Summation and discrimination of gratings moving in opposite directions. Vision Res 20, 341-347 (1980).

31. Wilson, H. R. \& Bergen, J. R. A four mechanism model for threshold spatial vision. Vision Res 19, 19-32 (1979).

32. Meese, T. S., Georgeson, M. A. \& Baker, D. H. Binocular contrast vision at and above threshold. J Vis 6, 1224-1243 (2006).

33. Meese, T. S. Spatially extensive summation of contrast energy is revealed by contrast detection of micro-pattern textures. J Vis 10, 14 1-21 (2010).

34. Tyler, C. W. \& Chen, C. C. Signal detection theory in the 2AFC paradigm: attention, channel uncertainty and probability summation. Vision Res 40, 3121-3144 (2000).

35. Meese, T. S. \& Williams, C. B. Probability summation for multiple patches of luminance modulation. Vision Res 40, 2101-2113 (2000).

36. Wilson, H. R., McFarlane, D. K. \& Phillips, G. C. Spatial frequency tuning of orientation selective units estimated by oblique masking. Vision Res 23, 873-882 (1983).

37. Georgeson, M. A. \& Shackleton, T. M. Perceived contrast of gratings and plaids: non-linear summation across oriented filters. Vision Res 34, 1061-1075 (1994).

38. Losada, M. A. \& Mullen, K. T. The spatial tuning of chromatic mechanisms identified by simultaneous masking. Vision Res 34, 331-341 (1994).

39. Vimal, R. L. Spatial-frequency tuning of sustained nonoriented units of the redgreen channel. J Opt Soc Am A 15, 1-15 (1998).

40. Meese, T. S. \& Summers, R. J. Neuronal convergence in early contrast vision: binocular summation is followed by response nonlinearity and area summation. J Vis 9, 7 1-16 (2009).

41. Legge, G. E. Binocular contrast summation--II. Quadratic summation. Vision Res 24, 385-394 (1984).

42. Morgenstern, Y. \& Elder, J. H. Local visual energy mechanisms revealed by detection of global patterns. J Neurosci 32, 3679-3696 (2012).

43. Pelli, D. G. On the relation between summation and facilitation. Vision Res 27, 119-123 (1987).

44. Wiesel, T. N. \& Hubel, D. H. Spatial and chromatic interactions in the lateral geniculate body of the rhesus monkey. J Neurophysiol 29, 1115-1156 (1966).

45. Shapley, R. \& Hawken, M. J. Color in the cortex: single- and double-opponent cells. Vision Res 51, 701-717 (2011)

46. Conway, B. R. \& Livingstone, M. S. Spatial and temporal properties of cone signals in alert macaque primary visual cortex. J Neurosci 26, 10826-10846 (2006).

47. Johnson, E. N., Hawken, M. J. \& Shapley, R. The orientation selectivity of colorresponsive neurons in macaque V1. J Neurosci 28, 8096-8106 (2008).

48. Livingstone, M. S. \& Hubel, D. H. Anatomy and physiology of a color system in the primate visual cortex. J Neurosci 4, 309-356 (1984).

49. Thorell, L. G., De Valois, R. L. \& Albrecht, D. G. Spatial mapping of monkey V1 cells with pure color and luminance stimuli. Vision Res 24, 751-769 (1984).

50. Anderson, S. J. \& Burr, D. C. Spatial and temporal selectivity of the human motion detection system. Vision Res 25, 1147-1154 (1985). 
51. Kim, Y. J., Gheiratmand, M. \& Mullen, K. T. Cross-orientation masking in human color vision: application of a two-stage model to assess dichoptic and monocular sources of suppression. J Vis 13, 15 1-14 (2013).

52. Schluppeck, D. \& Engel, S. A. Color opponent neurons in V1: a review and model reconciling results from imaging and single-unit recording. J Vis 2, 480-492 (2002).

53. Friedman, H. S., Zhou, H. \& von der Heydt, R. The coding of uniform colour figures in monkey visual cortex. J Physiol 548, 593-613 (2003).

54. Hass, C. A. \& Horwitz, G. D. V1 mechanisms underlying chromatic contrast detection. J Neurophysiol 109, 2483-2494 (2013).

55. Cavina-Pratesi, C., Kentridge, R. W., Heywood, C. A. \& Milner, A. D. Separate channels for processing form, texture, and color: evidence from FMRI adaptation and visual object agnosia. Cereb Cortex 20, 2319-2332 (2010).

56. Essock, E. A. The oblique effect of stimulus identification considered with respect to two classes of oblique effects. Perception 9, 37-46 (1980).

57. Cole, G. R., Hine, T. \& Mcllhagga, W. Detection mechanisms in L-, M-, and Scone contrast space. J Opt Soc Am A 10, 38-51 (1993).

58. Sankeralli, M. J. \& Mullen, K. T. Estimation of the L-, M- and S-cone weights of the post-receptoral detection mechanisms. J Opt Soc Am A 13, 906-915 (1996).

59. Wichmann, F. A. \& Hill, N. J. The psychometric function: I. Fitting, sampling, and goodness of fit. Percept Psychophys 63, 1293-1313 (2001).

60. Wichmann, F. A. \& Hill, N. J. The psychometric function: II. Bootstrap-based confidence intervals and sampling. Percept Psychophys 63, 1314-1329 (2001).

\section{Acknowledgments}

We thank Mark Georgeson and Tim Meese for their suggestions on fitting and modeling in early discussion of this work. Also we thank our subjects for participating in this study. This research was supported by a Canadian Institutes of Health Research (CIHR) grant MOP10819 to K.T.M.

\section{Author contributions}

M.G. and K.M. conceived the experiments. M.G. ran the experiments, programmed the models and analyzed the data. M.G. and K.M. wrote the manuscript.

\section{Additional information}

Supplementary information accompanies this paper at http://www.nature.com/ scientificreports

Competing financial interests: The authors declare no competing financial interests.

How to cite this article: Gheiratmand, M. \& Mullen, K.T. Orientation tuning in human colour vision at detection threshold. Sci. Rep. 4, 4285; DOI:10.1038/srep04285 (2014).

(c) (i) (2) This work is licensed under a Creative Commons Attribution-

BY NC SA NonCommercial-ShareAlike 3.0 Unported license. To view a copy of this license, visit http://creativecommons.org/licenses/by-nc-sa/3.0 